\title{
Relación entre síntomas psicopatológicos y calidad de vida en mujeres y hombres
}

\author{
Relationship between psychopathological symptoms and quality \\ of life in women and men
}

\author{
Ana Olivia Ruiz Martínez ${ }^{\mathrm{a}, *}$, Norma Ivonne González Arratia López Fuentes ${ }^{\mathrm{a}}$, Sergio \\ González-Escobara, Yessica Paola Aguilar Montes de Oca ${ }^{a}$, Martha Adelina Torres Muñoz \\ aUniversidad Autónoma del Estado de México, México
}

\section{Resumen}

Los problemas de salud mental afectan el desarrollo biopsicosocial de las personas. Dichos problemas empeoran debido a que se acude tardíamente a los servicios de salud, evidenciando la necesidad de detecciones tempranas en población abierta. Por ello, el objetivo de esta investigación fue evaluar la relación entre síntomas psicopatológicos y calidad de vida en mujeres y hombres. Participaron 465 personas, habitantes del Estado de México, quienes contestaron el SCL-90-R y el WHOQOL-BREF. En los resultados, las mujeres presentan mayores puntuaciones de síntomas psicopatológicos y menor calidad de vida con respecto a los hombres. Los problemas de salud mental afectan la calidad de vida por diferentes vías de influencia de acuerdo al sexo; en las mujeres destaca la depresión y la sintomatología global; y en los hombres, la somatización, la sintomatología global y la depresión. Se discute sobre la necesidad de prevención y detección precoz de los síntomas incipientes a través del trabajo comunitario.

Palabras clave: salud mental, calidad de vida, género, depresión, somatización.

\begin{abstract}
Mental health problems, which affect biopsychosocial development of people, worsen because people take too long to come to health services. This situation shows the need for early detection of these problems in open populations. Considering this, the present research aimed to assess the relationship between psychopathological symptoms and quality of life in women and men. The study population consisted of 465 inhabitants of the State of Mexico, who answered the SCL-90-R and WHOQOLBREF questionnaires. Results: women have higher scores of psychopathological symptoms and lower quality of life than men. Mental health problems affect quality of life in different ways according to gender: in women, depression and global symptoms stand out; and in men, somatization, global symptoms and depression are the most common ones. This paper discusses the need for prevention and early detection of incipient symptoms of mental health problems through community work.
\end{abstract}

Keywords: mental health, quality of life, gender, depression, somatization.
Para citar este artículo:

Ruiz, A., González Arria, N., González-Escobar, S., Aguilar, Y., \& Torres, M. (2018). Relación entre síntomas psicopatológicos y calidad de vida en mujeres y hombres. Liberabit, 24(1), 97-113. doi: 10.24265/ liberabit.2018.v24n1.07
Este es un artículo Open Access bajo la licencia Creative Commons Atribución-NoComercial-CompartirIgual 4.0

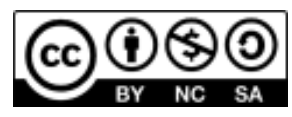




\section{Introducción}

El complejo proceso de salud-enfermedad oscila entre el bienestar y el malestar que experimentan las personas. Cuando la balanza se inclina hacia el extremo de la enfermedad, el malestar impide tener un óptimo desarrollo biológico, psicológico y social, lo cual ocurre tanto en la salud física como en la salud mental. De manera particular, el presente estudio aborda los problemas de salud mental en población no clínica, por considerar que es un área especialmente descuidada en las acciones de salud comunitaria.

Los problemas de salud mental abarcan diferentes afectaciones cognitivas, afectivo-emocionales y conductuales. Este malestar psicológico puede manifestarse en síntomas que afectan el desempeño hasta alcanzar un malestar significativo e incluso disfuncionalidad, en este punto es posible que la persona pueda ser ubicada bajo la categoría diagnóstica de alguna enfermedad o trastorno mental (Rodríguez, 2009). Estas problemáticas afectan a un porcentaje considerable de la población mundial. En América Latina y el Caribe se calcula que las personas presentan a lo largo de su vida las siguientes tasas en promedio: $11.3 \%$ abuso/dependencia de alcohol, 9.8\% depresión mayor y $5.6 \%$ de ansiedad generalizada (Kohn et al., 2005). Lo cual se agrava si se toma en cuenta la brecha del tratamiento, que representa la diferencia entre la prevalencia real de un trastorno y la presencia tratada, que en los países en desarrollo oscila entre el 76\% y el 85\% (Demyttenaere et al., 2004; Organización Mundial de la Salud [OMS], 2004).

En México, los trastornos con mayor frecuencia en población general se dan por ansiedad (14.3\%), consumo de sustancias (9.2\%) y afectivos (9.1\%) (Medina-Mora et al., 2003). Los hombres presentan mayor prevalencia de algún trastorno mental alguna vez en la vida destacando los trastornos relacionados con abuso de sustancias; en comparación con las mujeres quienes presentan mayor prevalencia en los últimos doce meses, sobresaliendo trastornos del estado de ánimo y ansiedad (Medina-Mora et al., 2005).

Mediante entrevista clínica en población comunitaria, se ha detectado que los trastornos mentales más incapacitantes pertenecen al espectro ansioso y depresivo (Saarni et al., 2007); específicamente en México, se reportan: estrés postraumático, fobia social y depresión. Las afectaciones de los problemas de salud mental por si solos, pueden ser mayores que las asociadas a las enfermedades crónicas como diabetes, hipertensión o artritis (Lara, Medina-Mora, Borges, \& Zambrano, 2007); más aún, la discapacidad aumenta cuando interactúan las enfermedades mentales y las crónicas (Scott et al., 2009). Por ejemplo, en pacientes con cáncer, las bajas puntuaciones en salud mental por sentirse desilusionados o tristes, reducen el tiempo de supervivencia (Reyes, Zhou, Ye, Wu, \& Hildebrandt, 2016); lo cual puede llegar a tal grado que le impida a la persona realizar totalmente sus actividades cotidianas y laborales (Alonso et al., 2011).

En este sentido, es importante destacar las afectaciones sobre la calidad de vida entendida como la percepción que tiene el individuo de su posición en la vida, dentro del contexto en el que vive, en relación con sus objetivos, estándares y preocupaciones (WHOQOL Group, 1997). Se reconoce que no existe un acuerdo unánime en su definición ni en los componentes que la integran; pero se coincide en señalar que se trata de un concepto multidimensional, que incluye tanto el bienestar subjetivo como las condiciones de vida objetivas, envueltos en un contexto sociocultural y medioambiental (Urzúa, 2012). Es por ello que se ha trabajado desde diferentes enfoques: biomédico, psicosocial, ecosistémico, conductual; así como con modelos específicos: satisfacción subjetiva, combinado (importancia-satisfacción), dinámico (sociocultural-personalidad) y funcionamiento del rol (Verdugo \& Martín, 2002). Dado su carácter multidimensional, se proponen algunas áreas como: salud física, estado psicológico, independencia, relaciones sociales, creencias personales y 
características del entorno; enfatizando que estas dimensiones interactúan entre sí y al momento de evaluarlas se deben considerar tanto aspectos positivos como negativos (Fernández \& Rojo, 2005).

El concepto de calidad de vida adquiere relevancia porque permite evaluar aspectos positivos del bienestar que experimentan las personas en diferentes esferas de su vida, validando la experiencia subjetiva y recuperando los recursos para la integración a la vida comunitaria. No se limita solo a curar lo psicopatológico (Verdugo \& Martín, 2002). En pacientes afectados por alguna enfermedad, suele trabajarse la Calidad de Vida Relacionada con la Salud (CVRS) que aborda la experiencia que tiene el paciente sobre su enfermedad, incluso se utiliza para evaluar los resultados del tratamiento y uso de servicios (Martín-Fernández et al., 2010). En cambio, en estudios de población abierta se utiliza la evaluación de la calidad de vida en general porque las personas no suelen sentirse enfermas, no cuentan con un diagnóstico, ni acuden a solicitar un servicio de salud; sino que se evalúa el bienestar cotidiano, sin enfermedad (Urzúa, 2010).

Las investigaciones en población clínica muestran que diferentes trastornos mentales (estado de ánimo, ansiedad, somatomorfos y alimentarios) y enfermedades físicas se asocian con el deterioro en la calidad de vida. Al identificar el trastorno que más contribuye al deterioro de la calidad de vida, se señala que la depresión afecta a todos los componentes de la calidad de vida, mientras que otros trastornos solo afectan ciertos dominios (Spitzer et al., 1995). El discurso de los pacientes indica que la depresión es el trastorno que más les impacta porque se mantiene presente en todo momento (Connell, O'Cathain, \& Brazier, 2014). Entre las dimensiones de la calidad de vida que se ven afectadas en los pacientes se encuentran: malestar, dificultades en sus relaciones sociales, disminución de actividades, falta de confianza en sí mismos y de autonomía, y deterioro en su salud. Aunque cabe señalar que también reconocen algunos aspectos positivos como la importancia del cuidado recibido, apoyo, esperanza y expectativas de mejoría. En este sentido, se reconoce que los pacientes con mayor severidad presentan mayor deterioro en la calidad de vida (Connell et al., 2014). Asimismo, se reporta el deterioro en la calidad de vida en población subclínica con síntomas ansiosos y depresivos (Spitzer et al., 1995). En el caso de la población no clínica, esta suele trabajarse como grupo de comparación de aquellos grupos con enfermedades físicas como diabetes, insuficiencia renal, cáncer, etc.; o con trastornos mentales de depresión, somatomorfos, alimentarios, etc. De esta manera, se presenta una tendencia favorable tanto en salud mental como en calidad de vida en comparación con los pacientes afectados. Sin embargo, existen pocos estudios en los que se valore a la población comunitaria como centro de interés, que permitan tener el conocimiento necesario sobre la gravedad y la carga que tienen los problemas de salud mental en el nivel de la población general (Saarni et al., 2007).

A pesar de las consecuencias negativas en la calidad de vida, las personas con problemas de salud mental suelen ser atendidas de forma tardía e insuficiente. La atención suele brindarse hasta que la persona ya presenta un trastorno mental. Esta demora se asocia con el desconocimiento que tienen las personas de los síntomas iniciales y las afectaciones incipientes, es decir, carecen de conocimiento para la detección; lo que se suma a la escasez de personal, servicios y acciones comunitarias sobre salud mental. Todo ello acarrea mayor severidad, comorbilidad, costo del tratamiento y peor pronóstico de recuperación; aumentando la brecha de tratamiento y deteriorando la calidad de vida. Estas carencias se acentúan en aquellas comunidades con menor grado de desarrollo, con desventajas sociales e inequidad (OMS, 2004), por lo que se requieren diagnósticos comunitarios que evalúen las necesidades y condiciones de cada contexto que sirvan como un marco de referencia para las intervenciones de promoción, prevención y detección oportuna (Saraceno \& Fleischmann, 2009). 
La evolución de los trastornos mentales indica que el malestar y los síntomas se presentan previamente al establecimiento del diagnóstico, también sabemos que algunas personas que lo requieren no asisten a los servicios de salud, es decir que en la población comunitaria ya están presentes algunos síntomas de los trastornos mentales y que no están siendo identificados. Sin embargo, desconocemos cuáles son los síntomas incipientes que se presentan con mayor frecuencia en la población comunitaria y de qué manera la presencia de estos síntomas psicopatológicos influye sobre diversas áreas de la calidad de vida de población adulta de comunidad abierta.

Dado lo anterior, el objetivo del presente estudio fue evaluar la relación entre los síntomas psicopatológicos y la calidad de vida en mujeres y hombres de población comunitaria. Esto con la intención de clarificar si los síntomas incipientes que generalmente no son diagnosticados en la vida cotidiana, tienen repercusiones en la calidad de vida de los habitantes de comunidad abierta.

\section{Método}

\section{Participantes}

Se trabajó con una muestra no probabilística, intencional, de sujetos voluntarios conformada por 465 personas de ambos sexos, 58.1\% mujeres $(n=270)$ y $41.9 \%$ hombres $(n=195)$; con edad promedio de $34.31(D E=13.19)$ años; de estado civil casados (43\%), solteros (35.1\%), de unión libre (15.6\%), viudos (2.2\%) y divorciados (2\%); con nivel educativo primaria (13.7\%), secundaria (36\%), bachillerato (25.4\%), universidad (18.4\%) y sin estudios (6.5\%); y, por último, habitantes de la región noreste del Estado de México, que al pertenecer a la misma área geodemográfica comparten de manera general, características étnicas, culturales y servicios de salud.

\section{Instrumentos}

Inventario de Síntomas (SCL-90-R). Auto-reporte que evalúa los síntomas psicopatológicos comunes (como somatizaciones, obsesiones y compulsiones, sensibilidad interpersonal, depresión, ansiedad, hostilidad, ideación paranoide y psicoticismo), así como la sintomatología global (Índice de Severidad Global, GSI). Consta de que el participante conteste 90 reactivos utilizando una escala de 5 opciones de respuesta (nada, muy poco, poco, bastante, mucho) para señalar cuáles son los síntomas que le han preocupado o molestado durante los últimos siete días. Fue creado por Derogatis (1977). Su versión reciente (Derogatis, 2002) fue adaptada a España por González de Rivera et al. (2002) y validada en México por Cruz, López, Blas, González y Chávez (2005). En este estudio se obtuvo un alfa de Cronbach de .98 y una varianza explicada de $64 \%$, en todas las dimensiones las puntuaciones más altas indican mayores síntomas.

WHOQOL-BREF. Escala de auto-reporte que evalúa la calidad de vida considerando cuatro componentes: salud física, salud psicológica, relaciones sociales y medio ambiente. Esta versión breve contiene 26 reactivos que se contestan con una escala de 1-5. Mayores puntuaciones indican mayor calidad de vida. Se interpretan tres niveles: superior, promedio e inferior. Elaborada por el grupo WHOQOL de la OMS (1997), cuya validación en México corresponde a González-Celis, Trón y Chávez (2009). En el presente estudio, se obtuvo un alfa de Cronbach de .91 y 54\% de varianza explicada en este instrumento.

\section{Procedimiento}

Dado que se trata de un trabajo de aplicación comunitaria, se capacitó a los aplicadores provenientes de la universidad estatal, cuidando que fueran pobladores de la misma área geográfica donde se recolectó la información. Ello con la intención de contar con personal familiarizado con la zona que pudiera identificarse con una institución oficial de la región con la intención de inspirar confianza en los participantes y así facilitar la recolección de datos directamente con población abierta.

Posteriormente se acudió a cada uno de los municipios, se identificaron los diferentes escenarios 
disponibles en la comunidad, como: domicilio particular, calle, escuelas, parques, comercios, mercados, centros de salud, hospitales, etc.; así mismo se identificó a personas mayores de 18 años, habitantes del municipio en cuestión, disponibles para participar voluntariamente. En dichos lugares, los aplicadores se acercaron personalmente a cada participante explicándole el proyecto y solicitando su participación, aquellos que aceptaron firmaron la carta bajo consentimiento informado.

Acto seguido los participantes contestaron los cuestionarios de manera individual; en aquéllos casos que se encontró dificultad para la lectura o de visión, los aplicadores leían y anotaban las respuestas del participante. Por último, se agradeció su participación.

En cuanto a las consideraciones éticas se trabajó en estricto apego a lo establecido en la Declaración de Helsinki, las normas éticas del APA y el Código Ético del Psicólogo, manteniendo en todo momento el respeto a la integridad, participación voluntaria y confidencialidad. Debido a que se trabajó con población abierta no se requirió la aprobación del comité de ética de alguna organización que permitiera el acceso a su población; por ello se trabajó directamente con el consentimiento informado que otorgaron los participantes de manera voluntaria.

\section{Análisis de datos}

De manera preparatoria, se compararon la edad ( $t$ Student) y la escolaridad (chi- cuadrado). Asimismo, se utilizaron las puntuaciones del SCL-90-R y del WHOQOL para realizar el análisis descriptivo (media, $D E$ ) y comparativo entre mujeres y hombres ( $t$ Student). Al encontrar diferencias significativas ( $p>$.05), el resto de los análisis se realizó de manera separada para mujeres y hombres. Posteriormente, se elaboraron correlaciones bivariadas entre cada uno de los síntomas y los elementos de calidad de vida. Por último, se utilizó la regresión lineal multivariada para generar modelos predictivos. Se utilizó el programa SPSS versión 23.

\section{Resultados}

En primer lugar, se realizó el análisis descriptivo y comparativo de las variables de edad y escolaridad. No se encontraron diferencias significativas en la edad de mujeres $(M=34.25, D E=12.70)$ y hombres $(M=34.42, D E=13.86)$, al obtener una $t=.15 \mathrm{y}$ sig. $=.88$. Tampoco se encontraron diferencias significativas en el nivel de escolaridad entre mujeres y hombres $\left(\chi^{2}=10.17, p=.07\right)$. Posteriormente, se analizaron los resultados descriptivos del SCL-90-R; las medias obtenidas mostraron que los principales síntomas en mujeres fueron: obsesión-compulsión, somatización y depresión; y en los hombres destacaron: obsesión-compulsión, ideación paranoide y somatización. Siguiendo a Grande, Newmeyer, Underwood y Williams (2014), se consideró el porcentaje de personas que presentaron una puntuación igual o mayor a una puntuación estándar superior a la media, encontrando que en las mujeres el porcentaje oscila entre $14.1 \%$ y $16.3 \%$; y en el caso de los hombres varían entre $6.7 \%$ al $14.4 \%$, identificando a las personas con alta frecuencia y severidad de los diferentes síntomas evaluados.

El análisis comparativo con las puntuaciones del SCL-90-R mostró diferencias significativas entre mujeres y hombres, por ello el resto de los análisis se realizaron por separado. En todos los síntomas evaluados, las mujeres obtuvieron mayores puntuaciones que los hombres. Asimismo, se compararon las puntuaciones de mujeres y hombres obtenidas en el WHOQOL-BREF encontrando diferencias significativas en todas las áreas evaluadas, donde las mujeres presentaron menor calidad de vida (Tabla 1). 
Tabla 1

Comparación de síntomas y calidad de vida entre mujeres y hombres

\begin{tabular}{|c|c|c|c|c|c|c|c|c|}
\hline \multirow{2}{*}{$\begin{array}{l}\text { Síntomas } \\
\text { SCL-90-R }\end{array}$} & \multicolumn{2}{|c|}{$\begin{array}{c}\text { Mujeres } \\
n=270\end{array}$} & \multicolumn{2}{|c|}{$\begin{array}{c}\text { Hombres } \\
n=195\end{array}$} & \multirow{2}{*}{$\begin{array}{c}\text { Comparación } \\
t \\
\end{array}$} & \multicolumn{2}{|c|}{$95 \%$ IC } & \multirow{2}{*}{$d$} \\
\hline & $M$ & $D E$ & $M$ & $D E$ & & LI & LS & \\
\hline Somatización & .71 & .70 & .42 & .50 & $5.23 * * *$ & .18 & .40 & .49 \\
\hline Obsesión-Compul. & .75 & .73 & .55 & .60 & $3.22 * * *$ & .08 & .32 & .30 \\
\hline Sensibilidad Interper. & .62 & .67 & .41 & .50 & $3.87 * *$ & .10 & .32 & .36 \\
\hline Depresión & .71 & .77 & .36 & .50 & $5.94 * * *$ & .23 & .46 & .56 \\
\hline Ansiedad & .61 & .67 & .37 & .53 & $4.26 * * *$ & .13 & .34 & .40 \\
\hline Hostilidad & .55 & .60 & .36 & .44 & $3.83 * * *$ & .09 & .28 & .36 \\
\hline Ansiedad Fóbica & .46 & .61 & .22 & .43 & $5.07 * * *$ & .15 & .34 & .47 \\
\hline Ideación paranoide & .67 & .75 & .46 & .60 & $3.49 * * *$ & .10 & .34 & .33 \\
\hline Psicotismo & .40 & .57 & .28 & .47 & $2.37 *$ & .02 & .21 & .22 \\
\hline GSI & .62 & .59 & .39 & .44 & $4.70 * * *$ & .13 & .32 & .44 \\
\hline \multicolumn{9}{|l|}{$\begin{array}{l}\text { Calidad de vida } \\
\text { WHOQOL }\end{array}$} \\
\hline Salud Física & 15.76 & 2.54 & 16.72 & 2.17 & $4.33 * * *$ & 1.39 & .52 & .41 \\
\hline Salud Psicológica & 15.10 & 2.83 & 16.24 & 2.39 & $4.69 * *$ & 1.62 & .66 & .44 \\
\hline Relaciones sociales & 15.30 & 3.28 & 16.10 & 2.70 & $2.94 * *$ & 1.37 & .27 & .28 \\
\hline Medio Ambiente & 12.76 & 2.26 & 13.96 & 2.28 & $5.6 * * *$ & 1.61 & .77 & .52 \\
\hline Calidad de Vida Total & 14.73 & 2.34 & 15.74 & 1.99 & $4.98 * * *$ & 1.44 & .62 & .23 \\
\hline
\end{tabular}

Nota: GSI = índice de severidad global; $M=$ media; $D E=$ desviación estándar; $t=t$ student; $* p<.05 ; * * p<.01 ; * * * p<.001 ;$ CI $=$ intervalo de confianza; LI = límite inferior; LS = límite superior; $d$ = magnitud del efecto.

En cuanto a la salud física, las mujeres perciben menor energía y se sienten menos hábiles y capaces para realizar sus actividades cotidianas y laborales; mientras que los hombres refieren mayor dolor físico y necesidad de tratamiento médico. Para ambos sexos, la satisfacción con su sueño es el aspecto con el que están menos satisfechos en esta área.

En la salud psicológica, las mujeres consideran que están insatisfechas y disfrutan menos la vida, aceptan menos su apariencia corporal y tienen menor capacidad de concentrarse; en tanto que los hombres tienen sentimientos negativos con mayor frecuencia. Ambos encuentran significado a su vida (religioso, espiritual o personal), y coinciden en que el aspecto más bajo es su capacidad para concentrarse.
En cuanto a las relaciones interpersonales, las mujeres están menos satisfechas con sus relaciones personales y sexuales que los hombres. Ambos consideran que cuentan con el apoyo de sus amigos, aunque para ellos es el aspecto con menor puntuación de esta área.

Con respecto al medio ambiente, las mujeres sienten menor seguridad y satisfacción con las condiciones en que viven y el ambiente que les rodea (información, servicios de salud, etc.) en comparación con los hombres. Ambos refieren una satisfacción media con los medios de trasporte y señalan como los aspectos más bajos: su participación en actividades recreativas y dinero suficiente para sus necesidades. 
Posteriormente, se evaluó la relación entre síntomas psiquiátricos y calidad de vida en cada sexo, encontrando relaciones negativas y significativas. En las mujeres se obtuvieron relaciones que oscilan entre $r=-.27$ y $r=-.62$; para los hombres las relaciones se encuentran entre $r=-.19$ y $r=-.49$ (Tabla 2).

Tabla 2

Correlaciones entre síntomas psicopatológicos y calidad de vida en mujeres y hombres

\begin{tabular}{llllll}
\multicolumn{1}{c}{ Calidad de Vida } & $\begin{array}{c}\text { Salud } \\
\text { Física }\end{array}$ & $\begin{array}{c}\text { Salud } \\
\text { Psicológica }\end{array}$ & $\begin{array}{c}\text { Relaciones } \\
\text { Sociales }\end{array}$ & $\begin{array}{c}\text { Medio } \\
\text { Ambiente }\end{array}$ & $\begin{array}{c}\text { CV } \\
\text { Total }\end{array}$ \\
\hline MUJERES & & & & & \\
\hline Somatización & $-.50^{* *}$ & $-.47^{* *}$ & $-.36^{* *}$ & $-.37^{* *}$ & $-.49^{* *}$ \\
Obsesión-Compulsión & $-.51^{* *}$ & $-.53^{* *}$ & $-.45^{* *}$ & $-.38^{* *}$ & $-.54^{* *}$ \\
Sensibilidad Interpers. & $-.44^{* *}$ & $-.50^{* *}$ & $-.44^{* *}$ & $-.38^{* *}$ & $-.51^{* *}$ \\
Depresión & $-.54^{* *}$ & $-.62^{* *}$ & $-.51^{* *}$ & $-.42^{* *}$ & $-.61^{* *}$ \\
Ansiedad & $-.48^{* *}$ & $-.50^{* *}$ & $-.36^{* *}$ & $-.34^{* *}$ & $-.49^{* *}$ \\
Hostilidad & $-.43^{* *}$ & $-.49^{* *}$ & $-.37^{* *}$ & $-.35^{* *}$ & $-.48^{* *}$ \\
Ansiedad Fóbica & $-.31^{* *}$ & $-.32^{* *}$ & $-.28^{* *}$ & $-.28^{* *}$ & $-.35^{* *}$ \\
Ideación Paranoide & $-.43^{* *}$ & $-.51^{* *}$ & $-.48^{* *}$ & $-.37^{* *}$ & $-.53^{* *}$ \\
Psicoticismo &.$- .48^{* *}$ & $-.55^{* *}$ & $-.44^{* *}$ & $-.37^{* *}$ & $-.54^{* *}$ \\
GSI & $-.54^{* *}$ & $-.59^{* *}$ & $-.49^{* *}$ & $-.42^{* *}$ & $-.60^{* *}$ \\
\hline HOMBRES & & & & & \\
\hline Somatización & $-.49^{* *}$ & $-.39^{* *}$ & $-.29^{* *}$ & $-.33^{* *}$ & $-.44^{* *}$ \\
Obsesión-Compulsión & $-.39^{* *}$ & $-.40^{* *}$ & $-.34^{* *}$ & $-.26^{* *}$ & $-.42^{* *}$ \\
Sensibilidad Interpers. & $-.31^{* *}$ & $-.38^{* *}$ & $-.38^{* *}$ & $-.28^{* *}$ & $-.41^{* *}$ \\
Depresión & $-.41^{* *}$ & $-.43^{* *}$ & $-.38^{* *}$ & $-.37^{* *}$ & $-.48^{* *}$ \\
Ansiedad & $-.34^{* *}$ & $-.35^{* *}$ & $-.30^{* *}$ & $-.25^{* *}$ & $-.37^{* *}$ \\
Hostilidad & $-.33^{* *}$ & $-.36^{* *}$ & $-.28^{* *}$ & $-.27^{* *}$ & $-.37^{* *}$ \\
Ansiedad Fóbica & $-.34^{* *}$ & $-.41^{* *}$ & $-.37^{* *}$ & $-.33^{* *}$ & $-.44^{* *}$ \\
Ideación Paranoide & $-.32^{* *}$ & $-.37^{* *}$ & $-.35^{* *}$ & $-.29^{* *}$ & $-.40^{* *}$ \\
Psicoticismo & $-.38^{* *}$ & $-.38^{* *}$ & $-.31^{* *}$ & $-.31^{* *}$ & $-.41^{* *}$ \\
GSI & $-.45^{* *}$ & $-.45^{* *}$ & $-.39^{* *}$ & $-.34^{* *}$ & $-.49^{* *}$ \\
\hline
\end{tabular}

Nota: GSI = índice de severidad global; ${ }^{* *} p<.01$

Por otro lado, se elaboraron los modelos predictivos para mujeres y hombres con la intención de identificar aquellos síntomas que tienen mayor impacto en la calidad de vida. Se utilizaron modelos de regresión lineal múltiple con el método por pasos, introduciendo como variables predictoras las puntuaciones obtenidas con el SCL-90-R y como variable dependiente las puntuaciones del WHOQOL. Se eligieron los modelos con menor colinealidad verificando que los valores de tolerancia y de inflación de la varianza (FIV) se ajustaran a 1000. El análisis de residuos se ajustó a la curva normal, con media = 0 y desviación estándar entre 1.70 y 2.74 .

En las mujeres, los síntomas depresivos y el Índice de Severidad Global (GSI) afectan las áreas específicas y el resultado total del instrumento de calidad de vida (WHOQOL-BREF). La depresión es 
la dimensión psicopatológica que tiene mayor efecto estadístico, incidiendo en dos áreas específicas: salud psicológica y relaciones sociales; así como en la calidad de vida total, explicando entre el $26 \%$ y el $38 \%$ de la varianza. Asimismo, la puntuación conjunta de los diversos síntomas, expresados en el GSI, inciden negativamente en la salud física y el medio ambiente, representando el $29 \%$ y el $17 \%$ de la varianza explicada sobre la calidad de vida (Tabla 3).

Tabla 3

Modelos predictivos de síntomas psicopatológicos sobre calidad de vida en mujeres

\begin{tabular}{llllll}
\hline \multicolumn{1}{c}{$\begin{array}{c}\text { Variable } \\
\text { Predictora }\end{array}$} & \multicolumn{1}{c}{$\begin{array}{c}\text { Variable } \\
\text { Dependiente }\end{array}$} & $R^{2}$ ajustada & $E E$ & $B$ & IC 95\% \\
\hline $\begin{array}{l}\text { Depresión } \\
\text { Depresión }\end{array}$ & Salud Psicológica & $.381^{* * *}$ & 2.23 & -.620 & -2.62 a -1.93 \\
Depresión & Calidad Vida Total & $.372 * * *$ & 1.85 & -.612 & -2.15 a -1.57 \\
GSI & Relaciones sociales & $.260 * * *$ & 2.80 & -.513 & -2.61 a -1.73 \\
GSI & Salud física & $.293^{* * *}$ & 2.14 & -.540 & -.77 a -1.90 \\
& Medio ambiente & $.175^{* * *}$ & 2.05 & -.422 & -2.02 a -1.19
\end{tabular}

Nota: $* * * p<.001$; Variables Predictoras = somatización, obsesiones-compulsiones, sensibilidad inter-personal, depresión, ansiedad, hostilidad, ansiedad fóbica, ideación paranoide y psicoticismo; GSI = índice de severidad global.

En cambio, en los hombres sobresalen la somatización, sintomatología total (GSI) y depresión como los elementos que influyen negativamente en la calidad de vida, explicando desde el $24 \%$ hasta el 13\% de la varianza del criterio. La somatización afecta la percepción de la salud física. La sintomatología total disminuye la calidad de vida total y dos áreas específicas: salud psicológica y relaciones sociales. Por último, los síntomas depresivos impactan sobre la percepción deteriorada del medio ambiente, aunque con una aportación menor: 13\% de la varianza explicada (Tabla 4).

Tabla 4

Modelos predictivos de síntomas psicopatológicos sobre calidad de vida en hombres

\begin{tabular}{llllll}
\hline \multicolumn{1}{c}{$\begin{array}{c}\text { Variable } \\
\text { Predictora }\end{array}$} & \multicolumn{1}{c}{$\begin{array}{c}\text { Variable } \\
\text { Dependiente }\end{array}$} & $R^{2}$ ajustada & $E E$ & $B$ & IC 95\% \\
\hline Somatización & Salud física & $.237^{* * *}$ & 1.89 & -.490 & -2.62 a -1.56 \\
GSI & Calidad Vida Total & $.234^{* * *}$ & 1.75 & -.487 & -2.78 a -1.64 \\
GSI & Salud Psicológica & $.200^{* * *}$ & 2.14 & -.452 & -3.15 a -1.76 \\
GSI & Relaciones sociales & $.145^{* * *}$ & 2.53 & -.387 & -3.23 a -1.59 \\
Depresión & Medio ambiente & $.129 * * *$ & 2.12 & -.366 & -2.22 a -1.04 \\
\hline
\end{tabular}

Nota: $* * * p<.001$; Variables Predictoras = somatización, obsesiones-compulsiones, sensibilidad inter-personal, depresión, ansiedad, hostilidad, ansiedad fóbica, ideación paranoide y psicoticismo; GSI = índice de severidad global. 


\section{Discusión}

La evaluación de la relación entre síntomas psicopatológicos y calidad de vida en población comunitaria, permitió identificar los síntomas más frecuentes en esta población; así como su impacto en la calidad de vida de hombres y mujeres. La descripción de los síntomas psicopatológicos en población comunitaria muestra que estos se presentan como malestares cotidianos, incomodidades y problemas atenuados. En la muestra estudiada, el porcentaje de personas que presentan puntuaciones altas de estos síntomas oscilan entre el 6\% y el 16\%, mostrando que la mayor parte de la población no presenta sintomatología. Esta tendencia coincide con los datos reportados en el ámbito internacional y nacional (Kohn et al., 2005; Medina-Mora et al., 2003, Saarni et al., 2007); aunque nuestros resultados resultan ligeramente mayores, al ser recolectados por un instrumento de tamizaje con menor especificidad que las entrevistas diagnósticas.

Estos síntomas se presentan con diferente frecuencia en hombres y mujeres. En todas las dimensiones evaluadas por el SCL-90-R, las mujeres obtuvieron puntuaciones más altas que los hombres, coincidiendo así con otros estudios en población clínica y comunitaria en el ámbito nacional e internacional (Rivera, Caballero, Pérez, \& Montero, 2013, Urbán et al., 2014) que señalan mayor psicopatología en el género femenino.

Los síntomas que se presentaron con mayor frecuencia en las mujeres fueron obsesiones y compulsiones, y somatización y depresión. Estas dimensiones resultan frecuentes en estudios con diversas poblaciones femeninas como pacientes clínicas, personal de salud (Cruz et al., 2005), estudiantes universitarios (Rivera et al., 2013), entre otras. De manera particular, nuestros datos se pueden comparar con el estudio de Lara, Espinosa, Cárdenas, Fócil y Cavazos (2005), ya que parte de su muestra corresponde a mujeres de población comunitaria mexicana. Los resultados promedio de depresión son iguales, aunque los nuestros son un poco mayores en obsesión-compulsión y somatización. Asimismo, coincidimos parcialmente con los resultados de Medina-Mora et al. (2005), quienes señalan que las mujeres presentan mayores síntomas de depresión y ansiedad; aunque con respecto al espectro ansioso en nuestra muestra, los datos elevados se obtuvieron en obsesivo-compulsivo más que ansiedad generalizada o ansiedad fóbica.

En el caso de los hombres, los síntomas más frecuentes fueron obsesión-compulsión, ideación paranoide y somatización. La literatura previa es consistente en la dimensión de obsesión-compulsión, pero presenta variabilidad en cuanto a las otras dos dimensiones. Los estudios con población masculina señalan que junto a obsesión-compulsión se presentan los síntomas depresivos, en tanto que somatización e ideación paranoide pueden estar elevados en algunas muestras pero en otras no (Cruz et al., 2005; González, Mercadillo, Graff, \& Barrios, 2007). Las puntuaciones obtenidas en nuestro estudio resultan ser menores que las de los otros estudios que incluyen hombres. Específicamente, la depresión alcanzó una puntuación inferior por lo que quedó ubicada entre los tres síntomas más disminuidos. Suponemos que esta tendencia a la baja puede relacionarse con las características de la muestra, en nuestro caso hombres adultos con escolaridad y ocupaciones diversas, es decir, personas no relacionadas con el ámbito clínico y con mayor heterogeneidad en sus datos; mientras que en otros estudios se ha trabajado con pacientes, familiares de pacientes, personal de salud, estudiantes y trabajadores universitarios conformando grupos homogéneos (Cruz et al., 2005; González et al. 2007; Rivera et al, 2013).

Estos resultados permiten apreciar que en la población comunitaria se encuentran presentes síntomas psicopatológicos que suelen pasarse por alto, tanto por la persona que los presenta como por el personal de salud. Además, se confirman las diferencias entre mujeres y hombres, siendo ellas las menos favorecidas al presentar mayores puntuaciones 
en los síntomas evaluados. Al respecto, una de las limitaciones del estudio es que no se evaluaron los síntomas relacionados con el consumo de sustancias, los cuales suelen ser más altos en los hombres; es por ello que nuestros resultados dejan un vacío considerable en este aspecto.

En cuanto a la calidad de vida, se encontró que los participantes indican, de manera general, que se sienten satisfechos con la vida. Las puntuaciones por área indican que la salud física es el aspecto que más incide sobre la calidad de vida, seguida por la salud psicológica, las relaciones sociales y, por último, el medio ambiente. El orden de las áreas es el mismo para hombres y mujeres, aunque se distinguen por la magnitud de sus puntuaciones. El análisis comparativo entre mujeres y hombres muestra diferencias en las puntuaciones obtenidas en todas las áreas evaluadas por el WHOQOL-BREF. Este indica que las mujeres refieren menor calidad de vida, ya que consideran que presentan frecuentemente menor rendimiento en sus actividades diarias, problemas emocionales e interpersonales, así como ligera insatisfacción con su medio ambiente. Nuestros resultados son contradictorios con lo encontrado en otros estudios, como el de Urzúa y Caqueo (2013), quienes al evaluar la calidad de vida en mujeres chilenas de población abierta reportaron mejores puntuaciones en salud física, psicológica y medio ambiente, en comparación con los hombres; lo que hace necesario que en futuros estudios se profundice sobre las variaciones conceptuales en diferentes regiones.

Con el estado actual del conocimiento, la tendencia indica que las mujeres de población general presentan menor calidad de vida en los dominios de salud física y salud psicológica en el ámbito internacional (Shevington, Lotfy, \& O'Connell, 2004). Esos resultados se extienden a población femenina universitaria, como muestra el estudio de Pagnin y de Queiroz (2015) con hombres y mujeres brasileños. En este sentido, Cruz, Polanczyk, Camey, Hoffmann y Fleck (2011) exploraron otras variables en población abierta, reportando que las menores puntuaciones en la calidad de vida (física, psicológica y medio ambiente), no solo se presentan en las mujeres sino también en otros subgrupos con menor nivel económico, menor escolaridad y con enfermedades crónicas.

La presencia de mayor sintomatología y menor calidad de vida en las mujeres puede estar asociada con un mayor reconocimiento de los síntomas en este género. Así también, puede estar asociada a las características socioculturales que las rodean en un país en desarrollo con predominio del modelo patriarcal, donde se interrelacionan algunos factores de riesgo tales como: roles de género tradicionales, desventajas socioeconómicas (menor sueldo y bajos puestos), discriminación, pobreza, etc. (Astbury, 2001).

En cuanto a la relación entre los síntomas detectados en población comunitaria con las dimensiones de la calidad de vida, los resultados demostraron la relación entre todos los síntomas y todas las dimensiones de la calidad de vida, tanto en mujeres como en hombres; aunque estas últimas mostraron una relación negativa más acentuada. De esta manera se reafirma que a mayor psicopatología, menor calidad de vida, lo que ocurre con mayor magnitud en las mujeres que en los hombres. Estas asociaciones han sido ampliamente documentadas en población clínica, con personas que presentan tanto enfermedades físicas como trastornos mentales, pero existe menor investigación centrada en personas de población abierta (Saarni et al., 2007), ya que este tipo de población suele utilizarse como eje de comparación. Así que con estos resultados se muestra que incluso la población comunitaria puede presentar problemáticas incipientes que requieren intervención psicológica.

Estas asociaciones entre las variables estudiadas alcanzan el nivel predictivo, donde aspectos como: depresión, sintomatología global y somatización repercuten sobre distintas áreas de la calidad de vida, con diferente magnitud de acuerdo al sexo de los participantes. 
En el caso de las mujeres, los síntomas depresivos y la sintomatología total fueron las variables predictivas. Los síntomas depresivos como sentirse decaídas, tristes o con pensamientos negativos son los aspectos que más disminuyen la calidad de vida, superando incluso los coeficientes de determinación de la sintomatología global (GSI). De manera específica, los síntomas depresivos incidieron sobre la calidad de vida total y sus componentes: salud psicológica y relaciones interpersonales; mostrando que hay un impacto en la satisfacción general con la vida y de manera particular una afectación en el área afectivo-emocional y la relación con otras personas significativas.

De esta manera, se concuerda con la tendencia internacional en cuanto a la sintomatología depresiva en mujeres, aunque en nuestros datos no se corrobora el impacto de la ansiedad. Esto podría deberse a nuestra forma de evaluación de este constructo, ya que el instrumento utilizado desglosa el espectro ansioso en diferentes dominios (ansiedad, obsesióncompulsión y ansiedad fóbica), de los cuales solo las obsesiones-compulsiones se presentaron con mayor frecuencia. Quizá la división de los síntomas evaluados contribuyó a que se diluyera el efecto.

En cuanto a la sintomatología depresiva en mujeres, desde hace tiempo se han escudriñado distintas formas de explicar esta tendencia. Al respecto, Afifi (2007) retoma las aportaciones de diversos autores que explican variables asociadas a la depresión. Por ejemplo, Kessler et al. (1994) consideran que el proceso de socialización permite la expresión de descontento y tristeza en las mujeres, facilitando la identificación de los síntomas depresivos; Nazroo, Edwards y Brown (1998) señalan que las mujeres se hayan expuestas a estresores relacionados con sus funciones reproductivas, la crianza de sus hijos y vivienda, en cambio los hombres señalan estresores financieros, laborales y conyugales.

En este sentido, Dalgard et al. (2006) indican que tanto hombres como mujeres de población comunitaria tienen eventos de vida estresantes aunque las mujeres tienen mayor soporte social; por lo que el impacto en los síntomas depresivos estaría dado por un efecto de interacción en aquellas mujeres que carecen de soporte social y presentan eventos negativos como: daño a parientes cercanos, problemas con amigos y crisis financiera. Estos también se constituyen como factores de riesgo para episodios recurrentes de depresión en población clínica femenina (van Loo, Aggen, Gardner, \& Kendler, 2015). Además, se reconoce que en la comprensión de la depresión se entretejen un sinnúmero de factores que requieren mayor investigación para lograr intervenciones oportunas y efectivas ante una problemática que sigue avanzando especialmente en población femenina.

En los hombres también destacaron la depresión y la sintomatología total (GSI), además se agrega la somatización como predictor de menor salud física. La sintomatología total es el aspecto que afecta más elementos de la calidad de vida, es decir que se requiere de la conjunción de síntomas para generar un impacto en los elementos evaluados: calidad de vida, salud psicológica y relaciones sociales. En cambio, la somatización solo impactó negativamente en la insatisfacción con la salud física y la depresión en el medio ambiente. Tanto la somatización como la depresión en población masculina requieren ser estudiadas con mayor profundidad, dado que en ambos se encuentran dificultades en la regulación emocional; además, en la somatización se suma la hipersensibilidad física (Sánchez-García, 2013). Por consiguiente, se observa que sí existe el impacto de la sintomatología sobre la calidad de vida de los hombres aunque en menor medida que en las mujeres.

En cuanto a la sintomatología global (GSI), se muestra su influencia tanto en hombres como mujeres, coincidiendo con estudios comunitarios como el de Saarni et al. (2007), quien señala que la sintomatología total genera menor calidad de vida relacionada con la salud, afectando aspectos como: estrés, vitalidad y sueño. Sin embargo, nuestros resultados muestran que 
la sintomatología global afecta áreas diferentes en hombres y mujeres, y que en estas últimas, la depresión rebasa el impacto de la sintomatología global.

Llama la atención que el impacto de la depresión sobre la calidad de vida y sus componentes no se muestra con la misma magnitud en los hombres; sino que en ellos se requiere de la conjunción de síntomas diversos para impactar en las mismas áreas, en cambio, la depresión solo incidió en la visión deteriorada del medio ambiente. En este sentido, otros estudios de mayor profundidad han señalado que los pacientes (hombres y mujeres) consideran que la depresión es lo que más les afecta porque los acompaña en todo momento: aumenta sus malestares y dificultades en diversas áreas mermando su funcionalidad y autonomía, por lo que disfrutan menos su vida (Connell et al., 2014). Sin embargo, esto solo coincide con nuestros resultados femeninos al impactar en casi todas las áreas de la calidad de vida, mientras que en el caso de los hombres el efecto es pequeño sobre la satisfacción con su entorno. Posiblemente esto se deba a que en población comunitaria los síntomas son incipientes y los hombres suelen solicitar ayuda especializada hasta que aumenta la gravedad (Gagne, Vasiliadis, \& Préville, 2014). Sin embargo, la direccionalidad de esta interpretación debe seguirse explorando, ya que algunos autores señalan que la dirección puede ser inversa. Por ejemplo, Hajek et al. (2015) evaluaron la evolución de la depresión y la calidad de vida relacionada con la salud mediante una encuesta con adultos mayores en Alemania, reportando qué cambios iniciales en la calidad de vida se acompañan de cambios posteriores en la depresión, principalmente en las mujeres, pero no se encontró asociación entre depresión inicial y cambios posteriores en la calidad de vida; evidenciando así que en estudios transversales como el presente, está intrínseca la dificultad de la predicción y la direccionalidad.

Las diferencias de género en los síntomas psicopatológicos y la calidad de vida de la población comunitaria, nos hace reflexionar sobre el papel de la mujer en la sociedad, entendiendo que esta desigualdad en la salud mental y la calidad de vida, refleja las inequidades de todo un ecosistema lleno de desigualdades económicas, políticas y socioculturales que permean desde el sistema individual hasta el sistema global. Donde los determinantes de la salud y roles tradicionales son marcadamente desiguales entre hombres y mujeres; así como en personas ubicadas en diferentes estratos socioeconómicos, constituyéndose como factores de riesgo que se acumulan y multiplican la disparidad de género.

Con lo expuesto, se puede concluir que en la población abierta existen síntomas incipientes, manifestaciones tempranas, malestares cotidianos que pueden deteriorar la calidad de vida, es decir que la relación entre estos aspectos no se circunscribe únicamente a la población clínica afectada por alguna enfermedad física o algún trastorno metal. Sino que esta asociación también ocurre en personas con síntomas incipientes, no detectados, que permanecen en sus comunidades sin identificar sus problemáticas ni comprender su relación con el deterioro de su calidad de vida, reflejado en menor bienestar físico, psicológico y social. Estas personas se beneficiarían de servicios escalonados de atención de salud mental en el primer nivel de atención, donde el médico general establece el enlace con el equipo de salud: enfermera psiquiátrica o psicólogo, quienes ofrecen una intervención inicial y comunicación constante con el médico y el psiquiatra. En caso de que la respuesta no sea favorable, entonces se refieren a los servicios especializados de salud mental (van Orden, Leone, Haffmans, Spinhoven, \& Hoencamp, 2017).

Los modelos realizados con población comunitaria muestran que los síntomas psicopatológicos afectan negativamente las diferentes áreas de la calidad de vida y que los síntomas siguieron vías de influencia diferentes de acuerdo al género, aunque los efectos predictivos son bajos sobretodo en el grupo de los hombres. 
$\mathrm{Al}$ retomar las aportaciones del presente estudio, deben considerarse las limitaciones en cuanto al muestreo no probabilístico, la falta de evaluación de problemas relacionados al consumo de sustancias y el diseño transversal que deja cuestionamientos por resolver. No obstante, se espera que esta aproximación de los síntomas en población comunitaria, nos lleve a comprender que este sector tiene necesidades propias que deben ser analizadas, más allá de su utilidad como grupo de comparación, exhortándonos a desarrollar detecciones oportunas e intervenciones eficaces de promoción sobre salud mental, prevención de trastornos mentales o atención clínica escalonada, considerando la participación activa de la comunidad desde una perspectiva de equidad de género.

\section{Conflicto de intereses}

Los autores manifiestan que no existen conflictos de intereses morales, económicos, laborales, de investigación, ni de cualquier otro tipo.

\section{Responsabilidad ética}

En el presente estudio no se han realizado experimentos en seres humanos, ni animales.

\section{Confidencialidad de los datos}

En el presente estudio se ha cuidado que los participantes voluntarios no corran ningún riesgo ni daño, Tal como ha quedado registrado ante la Secretaría de Investigación de la UAEM. Asimismo se encuentra apegado a la Declaración de Helsinki, los lineamientos éticos de la APA, y lineamientos del Código Ético del Psicólogo (México), como se expresa en el consentimiento informado.

\section{Derecho a la privacidad y consentimiento informado}

En el presente estudio se respeta la privacidad de los datos de los participantes, conforme se indica en el aviso de privacidad de la UAEM y el consentimiento bajo información.

\section{Agradecimiento}

El presente estudio ha sido financiado por la Universidad Autónoma del Estado de México y por la Secretaria de Educación Pública. 


\section{Referencias}

Afifi, M. (2007). Gender differences in mental health. Singapore Medical Journal, 48(5), 385-391. Recuperado de http://smj.sma.org.sg/4805/4805ra1.pdf

Alonso, J., Petukhova, M., Vilagut, G., Chatterji, S., Heeringa, S., Üstün, T. B.,... Kessler, R. (2011). Days out of role due to common physical and mental conditions: results from WHO World Mental Health surveys. Molecular Psychiatry, 16(12), 1234-1246. doi: 10.1038/mp.2010.101

Astbury, J. (2001). Gender disparities in mental health. En Organización Mundial de la Salud. 54 Asamblea Mundial de la Salud. Asamblea llevada a cabo en Ginebra, Suiza. Recuperado de http://www.who.int/ mental_health/media/en/242.pdf

Connell, J., O’Cathain, A., \& Brazier, J. (2014). Measuring quality of life in mental health: Are we asking the right questions? Social Science y Medicine, 120, 12-20. doi: 10.1016/j.socscimed.2014.08.026

Cruz, F. C. S., López, B. L., Blas, G. C., González, M. L., \& Chávez, B. R. A. (2005). Datos sobre la validez y confiabilidad de la Symptom Check List 90 (SCL 90) en una muestra de sujetos mexicanos. Salud Mental, 28 (1), 72-81. Recuperado de http://revista salud mental.mx/index.php/salud_mental/article/view/1037

Cruz, L., Polanczyk, C., Camey, S., Hoffmann, J., \& Fleck, M. (2011). Quality of life in Brazil: normative values for the WHOQOL-BREF in a southern general population sample. Quality of Life Research, 20, 1123-1129. doi: 10.1007/s11136-011-9845-3

Dalgard, O. S., Dowrick, C., Lehtinen, V., VazquezBarquero, P. C., Wilkinson, G., Ayuso-Mateos, J. L., ... ODIN Group (2006). Negative life events, social support and gender difference in depression. A multinational community survey with data from the ODIN study. Social Psychiatry and Psychiatric Epidemiology, 41, 444-451. doi: 10.1007/s00127006-0051-5

Demyttenaere, K., Bruffaerts. R., Posada-Villa, J., Gasquet, I., Kovess, V., Lepine, J. P., ... WHO World Mental Health Survey Consortium (2004). Prevalence, severity, and unmet need for treatment of mental disorders in the World Health Organization World Mental Health Surveys. JAMA, 291(21), 2581-2590. doi:10.1001/jama.291.21.2581

Derogatis, L. R. (1977). SCL-90-R. Symptom Checklist90-R. Administration, Scoring and Procedures Manual. Minneapolis: National Computer System.

Derogatis, L. R. (2002). SCL-90-R Symptom Checklist90-R. Cuestionario de 90 síntomas. España: Pearson.

Fernández, M. F., \& Rojo, P. F. (2005). Calidad de vida y salud: planteamientos conceptuales y métodos de investigación. Territoris, 5, 117-135. Recuperado de https://www.raco.cat/index.php/Territoris/article/ viewFile/117017/147939

Gagne, S., Vasiliadis, H., \& Préville, M. (2014). Gender differences in general and specialty outpatient mental health service use for depression. BMC Psychiatry, 14, 135-146. doi: 10.1186/1471-244X-14-135

González, S. L., Mercadillo, R. E., Graff, A., \& Barrios, F. A. (2007). Versión Computarizada para la aplicación de Listado de Síntomas 90 (SCL90) y del Inventario de temperamento y Carácter (ITC). Salud Mental 30(4), 31- 40. Recuperado de http://www.revistasaludmental. mx/index.php/salud_mental/article/view/1177

González-Celis, R. A. L., Trón, A. R., \& Chávez, B. M. (2009). Evaluación de Calidad de Vida a través del WHOQOL en Población de Adultos Mayores en México. México: UNAM FES Iztacala.

González de Rivera, J. L., De las Cuevas, C., \& Rodríguez, P. F. (2002). El cuestionario de 90 síntomas. Adaptación española del SCL-90-R. Madrid: TEA Ediciones.

Grande, T. L., Newmeyer, M. D., Underwood, L. A., \& Williams, C.R. (2014). Path analysis of the SCL-90$\mathrm{R}$ : Exploring use in outpatient assessment. Measurement and Evaluation in Counseling and Development, 47(4), 271-290. doi: 10.1177/074817 5614538061

Hajek, A., Brettschneider, C., Ernst, A., Lange, C., Wiese, B., Prokein, J.,... Konig, H. (2015). Complex coevolution of depression and health-related quality of life in old age. Quality of Life Research, 24(11), 2713-2722. doi: 10.1007/s11136-015-1005-8 
Kessler, R. C., McGonagle, K. A., Zhao, S., Nelson, C. B., Hughes, M., Eshleman, S., ... Kerndler, K. S. (1994). Lifetime and 12-month prevalence of DSMIII-R psychiatric disorders in the United States. Archives of General Psychiatry, 51(1), 8-19. doi:10.1001/archpsyc.1994.03950010008002

Kohn, R., Levav, I., Caldas de Almeida. J. M., Vicente, B., Andrade, L., Caraveo-Anduaga, J. J., ... Saraceno, B. (2005). Los trastornos mentales en América Latina y el Caribe: asunto prioritario para la salud pública. Revista Panamericana de Salud Pública, 18(4/5), 229-240. Recuperado de https://scielosp.org/article/ rpsp/2005.v18n4-5/229-240/es/

Lara, C., Espinosa, I., Cárdenas. M., Fócil, M., \& Cavazos, J. (2005). Confiabilidad y validez de la SCL-90 en la evaluación de psicopatología en mujeres. Salud Mental, 28(3), 42-50. Recuperado de http://revistasa ludmental.mx/index.php/salud_mental/article/view/ 1052

Lara, C., Medina-Mora, M. E., Borges, G., \& Zambrano, J. (2007). Social cost of mental disorders: disability and workdays lost, results from the Mexican Survey of Psychiatric Epidemiology. Salud Mental, 30(5), 4-11. Recuperado de http://revistasaludmental.mx/index.php/ salud_mental/article/view/1184

Martín-Fernández, J., Gómez-Gascón, T., del CuraGonzález, M. I., Tomás-García, N., Vargas-Machuca, C., \& Rodríguez-Martínez, G. (2010). La calidad de vida relacionada con la salud como factor explicativo de la utilización de la consulta de medicina de familia: un estudio bajo el modelo conductual. Revista Española de Salud Pública, 84(3), 309-319. Recuperado de https://dialnet.unirioja.es/ejemplar/246160

Medina-Mora, M. E., Borges, G., Lara, C., Benjet, C., Blanco, J., Fleiz, C., ... Aguilar-Gaxiola, S. (2003). Prevalence of mental disorders and use of services: Results from the Mexican National Survey of Psychiatric Epidemiology. Salud Mental, 26(4), 116. Recuperado de http://revistasaludmental.mx/ index.php/salud_mental/article/view/956

Medina-Mora, M. E., Borges, G., Lara, C., Benjet, C., Blanco, J., Fleiz, C., ... Zambrano, J. (2005). Prevalence, service use, and demographic correlates or 12-month DSM-IV psychiatric disorders in Mexico: Results from the Mexican National Comorbidity Survey. Psychological Medicine, 35(12), 1773-1783. doi: 10.1017/S003329170500 5672

Nazroo, J. Y., Edwards, A. C., \& Brown, G. W. (1998). Gender differences in the prevalence of depression: artefact, alternative disorder, biology or roles? Sociology of Health y Illness, 20(3), 312-330. doi:10.1111/14679566.00104

Orden, M. van, Leone, S., Haffmans, J., Spinhoven, P., \& Hoencamp, E. (2017). Prediction of mental health services use one year after regular referral to specialized care versus referral to stepped collaborative care. Community Mental Health Journal, 53(39), 316-323. doi: 10.1077/s10597-016-0046-y

Organización Mundial de la Salud (2004). Promoción de la salud mental: conceptos, evidencia emergente, práctica: Informe compendiado. Ginebra, Suiza: OMS.

Pagnin, D., \& de Queiroz, V., (2015). Comparison of Quality of life between medical students and young general populations. Education for Health, 28(3), 209-212. doi: 10.4103/1357-6283.178599

Reyes, M., Zhou, Y., Ye, Y., Wu, X., \& Hildebrandt, M. (2016). Mental health-related quality of life assessment at diagnosis as a predictor for prognosis of colorectal cancer patients. Cancer Research, 76(14), 34-66. doi: 10.1158/1538-7445.AM2016-3466

Rivera, L. A., Caballero, S. N. P., Pérez, S. I. N., \& Montero, L. L. M. (2013). SCL-90 R: distrés psicológico, género y conductas de riesgo. Universitas Psychologica, 12(1), 105-118. Recuperado de http://revistas.jave riana.edu.co/index.php/revPsycho/article/view/879

Rodríguez, J. J. (2009). Salud mental en la comunidad ( $2^{\mathrm{a}}$. ed.). Washington D.C.: Organización Panamericana de la Salud.

Saarni, S., Suvisaari, J., Sintonen, H., Pirkola, S., Koskinen, S., Aromaa, A., \& Lönnqvist, J. (2007). Impact of psychiatric disorders on health-related quality of life: general population survey. British Journal of Psychiatry, 190, 326-332. doi: 10.1192/ bjp.bp.106.025106 
Sánchez-García, M. (2013). Procesos psicológicos en la somatización: la emoción como proceso. International Journal of Psychology and Psychological Therapy, 13(2), 255-270. Recuperado de http://www.ijpsy.com/ volumen13/num2/360/procesos-psicolgicos-en-lasomatizacin-ES.pdf

Saraceno, B., \& Fleischmann, A. (2009). La salud mental desde una perspectiva mundial. En J. J. Rodríguez (Ed.), Salud mental en la comunidad (pp.13-25). Washington D. C.: Organización Panamericana de la Salud.

Scott, K. M., von Korff, M., Alonso, J., Angermeyer, M. C., Brmet, E., Fayyad, J., ... Williams, D. (2009). Mental-physical comorbidity and its relationship with disability: results from the World Mental health Surveys. Psychological Medicine, 39(1), 33-34. doi: 10.1017/S0033291708003188

Shevington, S. M., Lotfy, M., \& O’Connell, K. A. (2004). The World Health Organization's WHOQOL-BREF quality of life assessment: Psychometric properties and results of the international field trial A Report from the WHOQOL Group. Quality of Life Research, 13, 299-310. doi: 10.1023/B:QURE.0000018486. 91360.00

Spitzer, R., Koneke, K., Linzer, M., Hahn, S, Williams, J., de Gruy, F., ... Davies, M. (1995). Health-Related Quality of Life in Primary Care Patients with Mental Disorders. Results from the PRIME-MD 1000 Study. JAMA, 274(19), 1511-1517. doi:10.1001/jama. 1995.03530190025030

Urbán, R., Kun, B., Farkas, J., Paksi, B., Kökönyei, G., Unoka, Z., ... Demetrovics, Z. (2014). Bifactor structural model of symptom checklists: SCL-90-R and Brief Symptom Inventory (BSI) in a non-clinical community sample. Psychiatry Research, 216(1), 146-154. doi: 10.1016/j.psychres.2014.01.027

Urzúa, M. A. (2010). Calidad de vida relacionada con la salud: Elementos conceptuales. Revista Médica de Chile, 138(3), 358-365. doi: 10.4067/S0034-9887 2010000300017

Urzúa, M. A. (2012). Calidad de vida: una revisión teórica del concepto. Terapia psicológica, 1(30). doi: 10.406 7/S0718-48082012000100006

Urzúa, M. A., \& Caqueo, U. A. (2013). Estructura factorial y valores de referencia del WHOQOL-BREF en población adulta chilena. Revista Médica de Chile, 141, 1541-1546. doi: 10.4067/S0034-9887201300 1200008

van Loo, H., Aggen, S. H., Gardner, C. O., \& Kendler, K. S. (2015). Multiple risk factors predict recurrence of major depressive disorder in women. Journal of Affective Disorders, 180, 52-61. doi: 10.1016/j.jad. 2015.03.045

van Orden, M., Leone, S., Haffmans, J., Spinhoven, P., \& Hoencamp, E. (2017). Prediction of mental health services use one year after regular referral to specialized care versus referral to stepped collaborative care. Communiy Ment Health J., 53(3), 316-323. doi: 10.1007/s10597-016-0046-y

Verdugo, M. A., \& Martín, M. (2002). Autodeterminación y calidad de vida en salud mental: dos conceptos emergentes. Salud Mental, 25(4), 68-77.

WHOQOL Group (1997). WHOQOL Measuring Quality of Life. Ginebra, Suiza: WHO. Recuperado de http:// www.who.int/mental_health/media/68.pdf 


\section{Ana Olivia Ruiz Martínez}

Universidad Autónoma del Estado de México (México)

Doctora en Psicología, orientación en psicología y salud, Investigadora Nacional (SIN 1), integrante del Cuerpo académico: Cultura y Personalidad. Publicaciones nacionales e internacionales. Línea de investigación: Salud psicológica y factores asociados, mediante temas como: Salud mental, trastornos alimentarios, obesidad y enfermedades crónicas; factores familiares y socioculturales.

ORCID: 0000-0002-9286-5946

Autor corresponsal: ana_olivi@yahoo.com.mx

Norma Ivonne González Arratia López Fuentes

Universidad Autónoma del Estado de México (México)

Miembro del Sistema Nacional de Investigadores Nivel 1, Perfil PRODEP y es líder del Cuerpo Académico Consolidado Cultura y Personalidad desde 2001. Publicación de artículos científicos nacionales e internacionales, autora de 5 libros sobre: Resiliencia, autoestima, Bienestar, Psicología y Salud e Investigación Cualitativa. Línea de investigación: cultura, personalidad y salud, así como resiliencia y psicología positiva en niños y adolescentes en contextos de vulnerabilidad social.

ORCID: 0000-0003-0497-119X

nigalf@yahoo.com.mx

Sergio González-Escobar

Universidad Autónoma del Estado de México (México)

Doctor en Investigación Psicológica. Profesor investigador, perfil PROMEP. Publicaciones nacionales e internacionales. Temáticas: Depresión, Autoestima, Ansiedad, Estudios de Género (Masculinidad-Feminidad), Pareja, Autoconcepto. Línea de investigación: Psicología Clínica, Psicología de la Salud, Cultura y Personalidad, Significado Psicológico.

ORCID: 0000-0001-6611-3890

sergioglz4@hotmail.com

Yessica Paola Aguilar Montes de Oca

Universidad Autónoma del Estado de México (México)

Doctora en Ciencias de la Salud, orientación en psicología y salud. Desarrolla investigaciones en Salud psicológica orientados hacia la apatía, emociones negativas, expectativas de vida y factores asociados en hombres y mujeres.

ORCID: 0000-0002-4427-4973

amarem_ypam@hotmail.com

\section{Martha Adelina Torres Muñoz}

Universidad Autónoma del Estado de México (México)

Doctora en Ciencias de la Salud, cuenta con investigaciones en somatización, personalidad, ansiedad, depresión, pareja, emociones y familia. Su línea de investigación está centrada en temas relacionados a la cultura, personalidad y salud.

nina_156ch@hotmail.com 